\title{
Preparation and Performance of Inorganic Heat Insulation Panel Based on Sepiolite Nanofibers
}

\author{
Fei Wang, ${ }^{1,2}$ Jinsheng Liang, ${ }^{1}$ Haifeng Liu, ${ }^{1}$ Xinhui Duan, ${ }^{1}$ \\ Qingguo Tang, ${ }^{1}$ and Huimin Liu ${ }^{1}$ \\ ${ }^{1}$ Institute of Power Source \& Ecomaterials Science, Hebei University of Technology, Tianjin 300130, China \\ ${ }^{2}$ Key Laboratory for Palygorskite Science and Applied Technology of Jiangsu Province, Huaiyin Institute of Technology, \\ Huaian 223003, China
}

Correspondence should be addressed to Fei Wang; wangfei36777@126.com

Received 9 November 2013; Accepted 9 December 2013; Published 17 February 2014

Academic Editor: Xiangyu Zhao

Copyright (C) 2014 Fei Wang et al. This is an open access article distributed under the Creative Commons Attribution License, which permits unrestricted use, distribution, and reproduction in any medium, provided the original work is properly cited.

\begin{abstract}
High efficiency and low cost thermal insulation energy saving panel materials containing sepiolite nanofibers were developed by means of the synergistic action of inorganic adhesive, curing agent, and hydrogen peroxide. The water soluble sodium silicate was used as inorganic adhesive, and the sodium fluorosilicate was chosen as curing agent. Moreover, appropriate amount of hydrogen peroxide was added in order to decrease the bulk density and improve the heat insulation performance of panel materials. The results showed that the synergistic action of inorganic adhesive, curing agent, and hydrogen peroxide could make thermal insulation energy saving panel materials have low bulk density and high mechanical performance, and the optimal process was as follows: $120^{\circ} \mathrm{C}$ of drying temperature, $1.6 \%$ of sodium silicate as inorganic adhesive, $12 \%$ of sodium fluorosilicate as curing agent in sodium silicate, and $2.5 \%$ of hydrogen peroxide. The thermal insulation energy saving panel materials as prepared could arrest heat transmission and resist external force effectively.
\end{abstract}

\section{Introduction}

The external thermal insulation compound system containing organic panel materials and the organic coating have become two of the most common methods of construction energy saving in recent years. The former has disadvantages of cumbersome construction, high cost, narrow construction scope, and hot-bridge formation [1-3]. Moreover, the organic materials process would not only exacerbate tensions in the energy supply, but also pollute the environment. The coating as thin-layer thermal insulation technique has been used in various regions. For example, space refractive heat insulation coating materials as ceramic coating materials have been developed by scientific and technical personnel in the National Aeronautics and Space Administration to solve the control problems for spacecraft in the process of heat transfer, which has the characteristics of efficient thermal insulation and high reflectivity, but the above mentioned coating materials have not been used extensively in the field of civil building due to their high cost [4-7]. At present, most of the buildings containing inorganic panel have little or no thermal insulation performance [8], and the research on the inorganic thermal insulation panel based on the mineral nanomaterials could improve the thermal insulation performance, which has been scarcely reported. Moreover, the application of natural mineral materials as raw materials will solve the serious environmental pollution caused by the process of organic materials.

Sepiolite as a kind of porous mineral material widely existing in nature has many nanometer level micropores and mesopores, which can play an active role as an additive for composite materials in many fields such as thermal insulation [9-12], and the sepiolite mineral materials have been applied in many fields [13-18]. Sepiolite is a kind of mineral materials in the form of fiber assemblies, leading to the limitation of usage [19-22]. Although the defibering 
treatment of sepiolite as a simple and cheap way to obtain mineral nanofibers is available for practical use, it has been less investigated except for a few exploratory researches involving the destruction of its fibrous micromorphology by ball milling and the use of expensive and sensitive agents [2226], which provides promising future for further research. In our previous research, sepiolite nanofibers have been prepared using dry method airflow defibering technique [21].

The objective of this work is to develop high efficiency, low cost thermal insulation energy saving panel materials containing sepiolite nanofibers by means of the synergistic action of inorganic adhesive, curing agent, and hydrogen peroxide. And their process and performance are also investigated.

\section{Experimental Section}

The raw sepiolite samples as one of natural fibrillar minerals were supplied by LB Nanomaterials Technology Co., Ltd. The sodium silicate as inorganic adhesive, sodium fluosilicate as curing agent, and hydrogen peroxide were supplied by Kewei Chemical Group Co., Ltd. The compounding ingredients were purchased from chemical stores. $500.00 \mathrm{~g}$ of natural sepiolite samples was added into $3500 \mathrm{~mL}$ of water solution containing $175 \mathrm{~mL}$ of hydrochloric acid. After that, the mixture was stirred at speed of $2000 \mathrm{rpm}$ for $2 \mathrm{~h}$ by high-speed dispersing machine, and then the sepiolite solid was separated from the admixture by vacuum filtering and washed several times with distilled water until chlorine ion was not tested with silver nitrate, and then the dried cake at $60^{\circ} \mathrm{C}$ was crushed and ground into powders using the 20 mesh sieves. Then the above acid purified sepiolite samples were superfined by jet mill grinding. The grinding room air pressure is controlled between 0.7 and $0.85 \mathrm{MPa}$, the bearing protection air pressure is $0.2 \mathrm{MPa}$, the wash air pressure is $0.15 \mathrm{MPa}$, and the feeding rate is $6 \mathrm{~kg} / \mathrm{h}[21]$. The thermal insulation energy saving panel materials containing sepiolite nanofibers were developed by means of the synergistic action of inorganic adhesive, curing agent, and hydrogen peroxide. $50.00 \mathrm{~g}$ of above sepiolite samples was added into $200 \mathrm{~mL}$ of water solution containing a given amount of sodium silicate as inorganic adhesive, and a given amount of sodium fluorosilicate as curing agent and hydrogen peroxide was added into the above mixture sequentially, and then the mixture was stirred at speed of $300 \mathrm{rpm}$ for $1 \mathrm{~h}$ by highspeed dispersing machine; then the above mixture was dumped into mold, and the thermal insulation energy saving panel materials were obtained after drying for $24 \mathrm{~h}$ at a specified temperature. The microstructure of the samples was observed by scanning electron microscopy (PhilipsXL30) at $25.0 \mathrm{kV}$ and $30 \mu \mathrm{A}$. The Fourier transform infrared spectrum (FTIR) was performed on a BRUKER-80V made in Germany using $\mathrm{KBr}$ discs. The thermal insulation effect was evaluated by thermal insulation effect testing device [27], and the mechanical performance was measured following GB/T 5486.2-2001 using a universal tensile testing machine (CMT-6104).

\section{Results and Discussion}

Figure 1 shows the variation of compressive strength values of panel materials containing sepiolite nanofibers after drying at different temperatures.

From Figure 1, we can see that the panel materials containing sepiolite nanofibers after drying at different temperatures have different compressive strength values. Among them, the panel materials containing sepiolite nanofibers show the obvious decrease of compressive strength after drying when temperature is lower than $120^{\circ} \mathrm{C}$, and the panel materials containing sepiolite nanofibers after drying at $120^{\circ} \mathrm{C}$ show the highest compressive strength value. When the temperature is higher than $120^{\circ} \mathrm{C}$, the compressive strength starts to reduce. Moreover, Figure 2 shows the variation of bulk density values of panel materials containing sepiolite nanofibers after drying at different temperatures.

From Figure 2, we can see that the panel materials containing sepiolite nanofibers after drying at different temperatures have the different bulk density values. Among them, the panel materials containing sepiolite nanofibers show the obvious decrease of bulk density after drying when temperature is lower than $120^{\circ} \mathrm{C}$. When the temperature is higher than $120^{\circ} \mathrm{C}$, the panel materials containing sepiolite nanofibers have almost consistent bulk density values. As shown in Figure 1, the panel materials containing sepiolite nanofibers after drying at temperature of $120^{\circ} \mathrm{C}$ have the highest compressive strength value. Therefore, $120^{\circ} \mathrm{C}$ is chosen as the optimal drying temperature value. The main reason for the above phenomenon lies in that many $\mathrm{Si}-\mathrm{OH}$ bonds in the sodium silicate aqueous solution are water soluble, and the dehydration condensation reaction between the hydroxyl groups is difficult to carry out at too low temperature [28, 29]. When the temperature is too high, the panel materials as prepared are easy to crack due to the high water evaporation speed and sepiolite fibers agglomeration, thus leading to the decrease of compressive strength. Therefore, $120^{\circ} \mathrm{C}$ is chosen as the optimal drying temperature value.

Figure 3 shows the variation of compressive strength values of panel materials containing sepiolite nanofibers after adding different amounts of sodium silicate as inorganic adhesive.

From Figure 3, it can be seen that the panel materials containing sepiolite nanofibers after adding different amounts of sodium silicate as inorganic adhesive have different compressive strength values. Among them, the panel materials containing sepiolite nanofibers indicate the obvious increase of compressive strength after adding different amounts of sodium silicate when the addition amount of sodium silicate is less than $1.6 \%$. When the addition amount of sodium silicate is more than $1.6 \%$, the panel materials containing sepiolite nanofibers have almost consistent compressive strength values. Moreover, Figure 4 shows the variation of bulk density values of panel materials containing sepiolite nanofibers after adding different amounts of sodium silicate as inorganic adhesive.

From Figure 4, it can be seen that the panel materials containing sepiolite nanofibers after adding different amounts of sodium silicate have different bulk density values. Among 


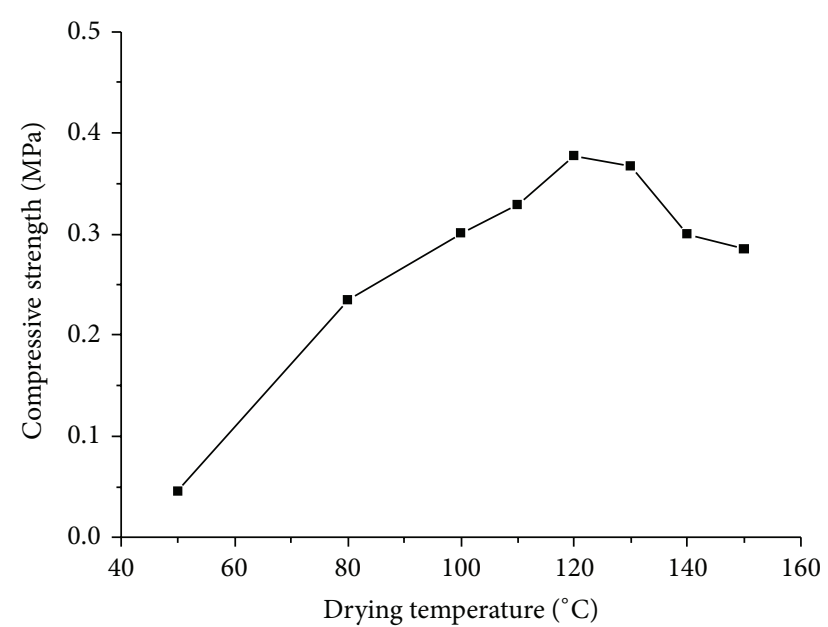

FIGURE 1: Variation of compressive strength values of panel materials containing sepiolite nanofibers after drying at different temperatures.

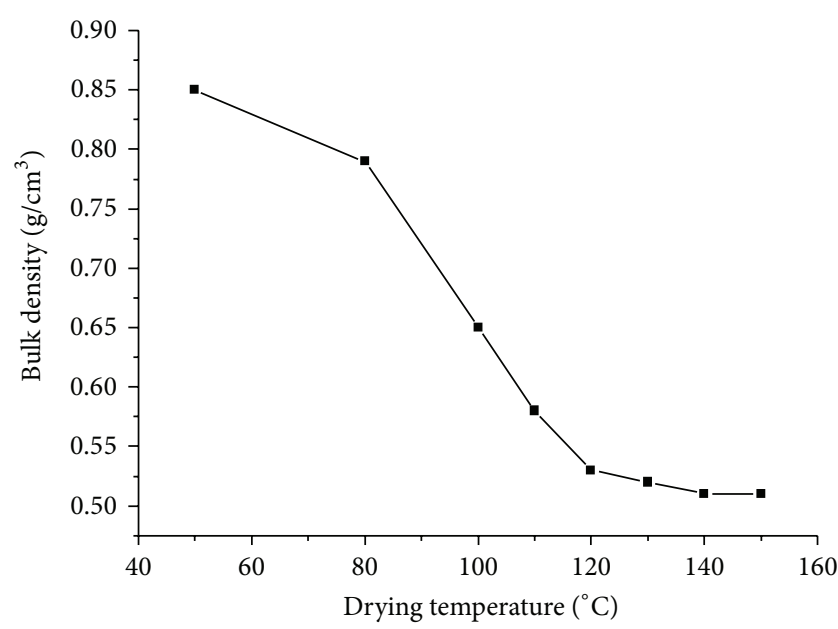

FIGURE 2: Variation of bulk density values of panel materials containing sepiolite nanofibers after drying at different temperatures.

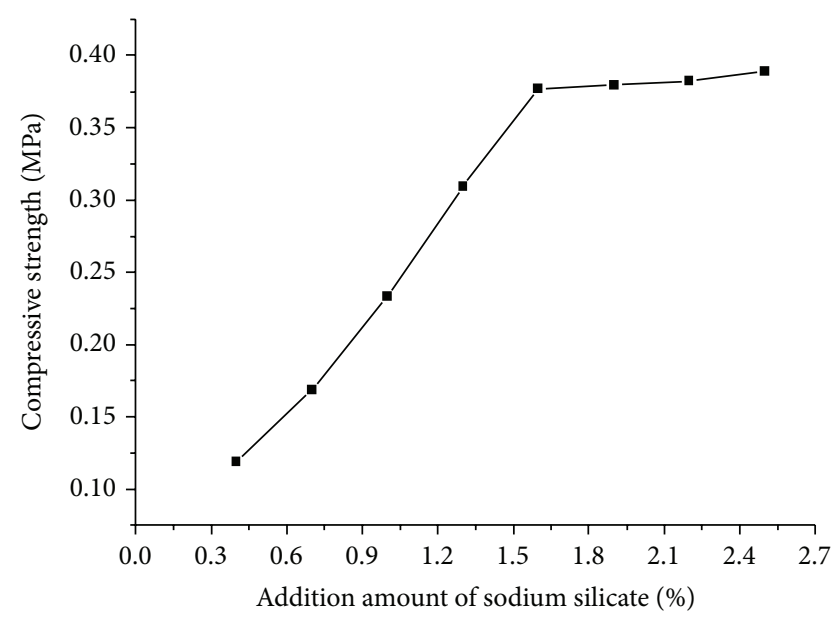

FIGURE 3: Variation of compressive strength values of panel materials containing sepiolite nanofibers after adding different amounts of sodium silicate as inorganic adhesive.

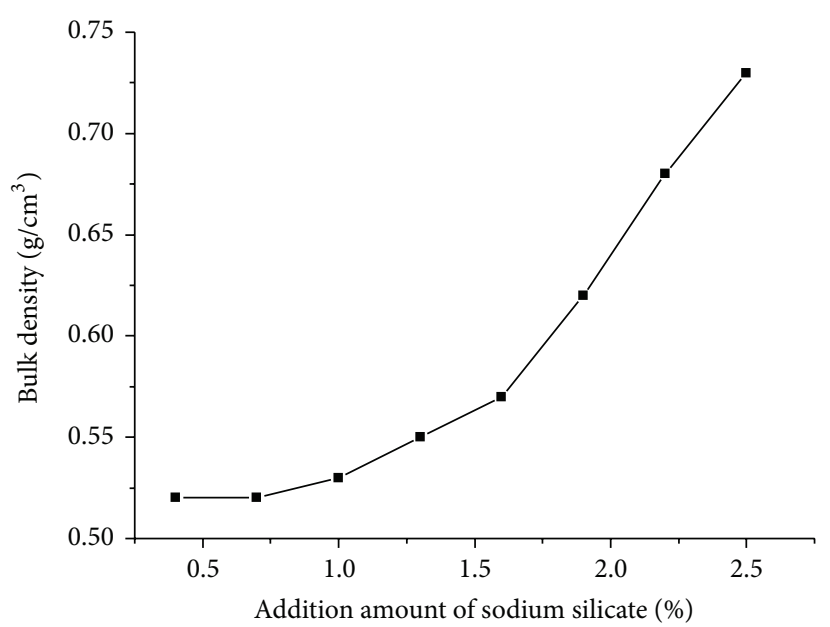

FIGURE 4: Variation of bulk density values of panel materials containing sepiolite nanofibers after adding different amounts of sodium silicate as inorganic adhesive.

them, the panel materials containing sepiolite nanofibers show the slight increase of bulk density after adding different amounts of sodium silicate when the addition amount of sodium silicate is less than $1.6 \%$. When the addition amount of sodium silicate is more than $1.6 \%$, the panel materials containing sepiolite nanofibers show significant increase of bulk density values. The main reason for this phenomenon is that sodium silicate as inorganic adhesive could make the compressive strength of panel materials containing sepiolite nanofibers increase obviously when the sodium silicate addition amount is less than 1.6\%; meanwhile the bulk density values of panel materials increase gradually. When the sodium fluorosilicate in sodium silicate addition amount is more than $1.6 \%$, the panel materials containing sepiolite nanofibers have almost consistent compressive strength values due to the requirement of sepiolite nanofibers adhesion. At the same time, the bulk density increase tendency of panel materials becomes more obvious because of the existence of excessive sodium silicate. According to the above analysis, $1.6 \%$ is chosen as the optimal condition of addition amount of sodium silicate. Moreover, the Fourier transform infrared spectrum (FTIR) of different sepiolite samples before and after reaction with sodium silicate is shown in Figure 5.

From Figure 5(a), it can be seen that FTIR spectrum of sepiolite samples before reaction with sodium silicate has many absorption peaks. The band at $3722 \mathrm{~cm}^{-1}$ is assigned to structural hydroxyls attached to silanol groups in sepiolite nanofiber, and the band at $3683 \mathrm{~cm}^{-1}$ is attributed to a hydroxyl on a magnesium ion located in the octahedral sheet. The band at $3570 \mathrm{~cm}^{-1}$ is assigned to bound water attached to magnesium ion located on the external channels of sepiolite. The 3420 and $1661 \mathrm{~cm}^{-1}$ bands are, respectively, assigned to the $\mathrm{OH}$ stretching and bending vibration, representing the zeolitic water in channels. From Figure 5(b, c), the Si-OH stretching vibration $\left(3722 \mathrm{~cm}^{-1}\right)$ disappears in the IR spectrum of sepiolite samples after reaction with sodium silicate, indicating the dehydration condensation reaction between 


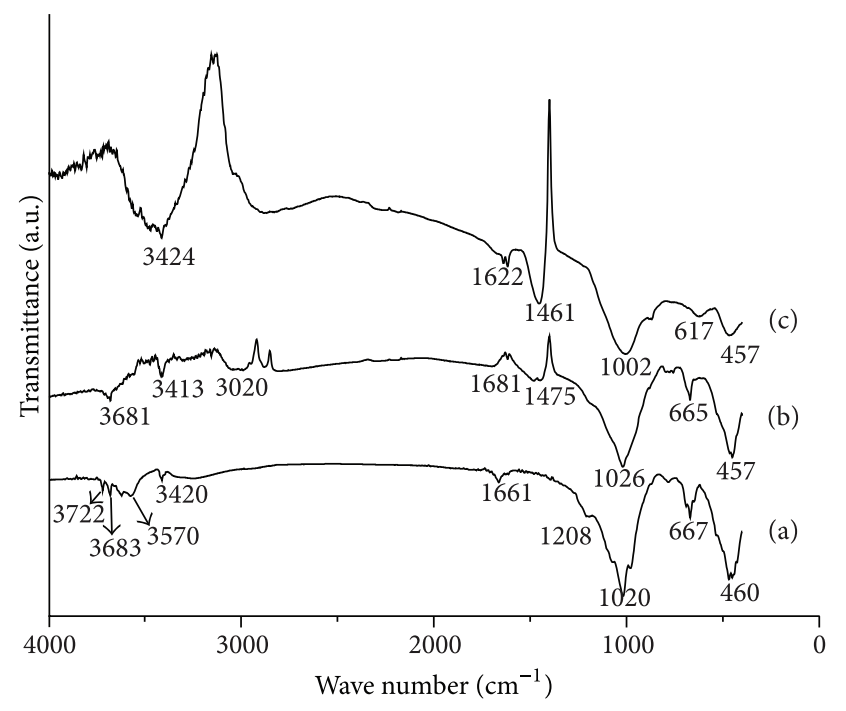

FIGURE 5: The Fourier transform infrared spectrum (FTIR) of different sepiolite samples before and after reaction with sodium silicate ((a) sepiolite samples before reaction with sodium silicate; (b) sepiolite samples after reaction with sodium silicate; (c) sodium silicate).

sodium silicate hydrolysis product and sepiolite nanofibers. The $3420 \mathrm{~cm}^{-1}$ band assigned to the $\mathrm{OH}$ stretching vibration of $\mathrm{Mg}_{3} \mathrm{OH}$ increases in intensity and shifts to $3413 \mathrm{~cm}^{-1}$, which is mainly caused by the formation of the $\mathrm{Si}-\mathrm{O}-\mathrm{Si}$ bonds in the sodium silicate hydrolysis product and sepiolite nanofibers. Furthermore the most bands mentioned above are not significantly changed, which reflects the structural stability of the sepiolite nanofibers during the above mentioned procedure [30-32].

Figure 6 shows the variation of compressive strength values of panel materials containing sepiolite nanofibers after adding different amounts of sodium fluorosilicate.

From Figure 6, it can be seen that the panel materials containing sepiolite nanofibers after adding different amounts of sodium fluorosilicate as curing agent have different compressive strength values. Among them, the panel materials containing sepiolite nanofibers show obvious increase of compressive strength after adding different amounts of sodium fluorosilicate when the addition amount of sodium fluorosilicate is less than $12 \%$ and it is up to maximum at $12 \%$ of addition amount of sodium fluorosilicate. When the addition amount of sodium fluorosilicate is more than $12 \%$, the compressive strength values of panel materials start to decrease gradually. Moreover, Figure 7 shows the variation of bulk density values of panel materials containing sepiolite nanofibers after adding different amounts of sodium fluorosilicate as curing agent.

From Figure 7, it can be seen that the panel materials containing sepiolite nanofibers after adding different amounts of sodium fluorosilicate have similar bulk density values. Among them, the panel materials containing sepiolite nanofibers show slight increase of bulk density after adding different amounts of sodium fluorosilicate. As shown in

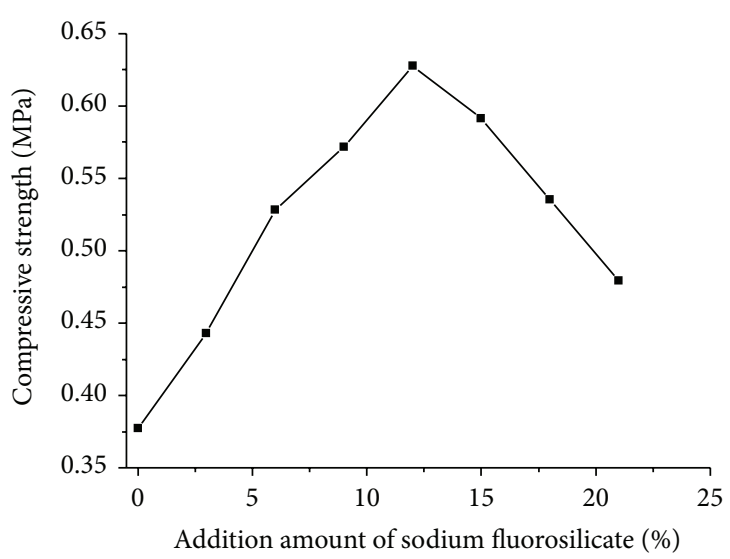

FIGURE 6: Variation of compressive strength values of panel materials containing sepiolite nanofibers after adding different amounts of sodium fluorosilicate in sodium silicate as curing agent.

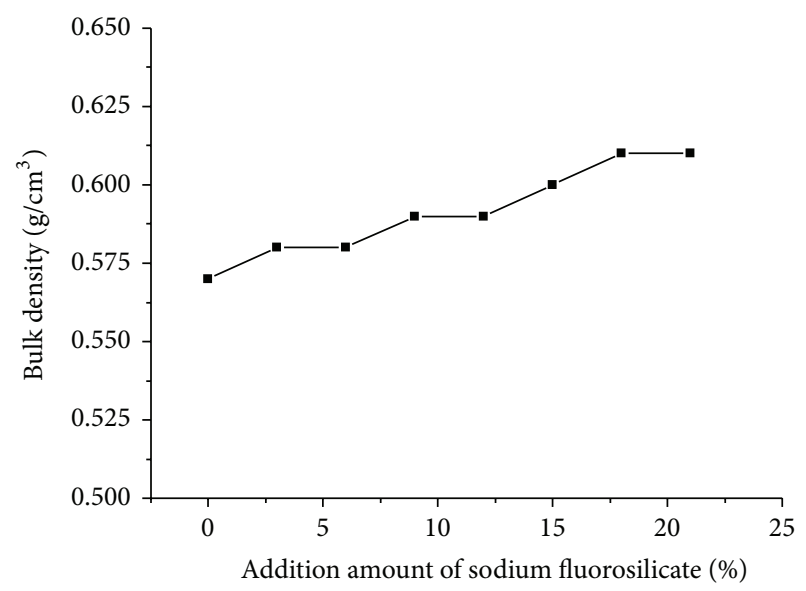

FIGURE 7: Variation of bulk density values of panel materials containing sepiolite nanofibers after adding different amounts of sodium fluorosilicate in sodium silicate as curing agent.

Figure 6, the panel materials containing sepiolite nanofibers after adding $12 \%$ of sodium fluorosilicate in sodium silicate as curing agent in sodium silicate have the best compressive strength. The main reason for this phenomenon is that sodium fluorosilicate as curing agent could make the compressive strength of panel materials containing sepiolite nanofibers increase gradually when the sodium fluorosilicate in sodium silicate addition amount is less than $12 \%$. When the sodium fluorosilicate addition amount is more than $12 \%$, the compressive strength of panel materials containing sepiolite nanofibers starts to decrease due to the too fast curing progress. According to the above analysis, we decide to choose $12 \%$ as the optimal condition of addition amount of sodium fluorosilicate in sodium silicate.

For the thermal insulation materials, the decrease of bulk density is helpful to improve the heat insulation properties of materials, under the prerequisite of maintaining a certain mechanical performance such as compressive strength of 


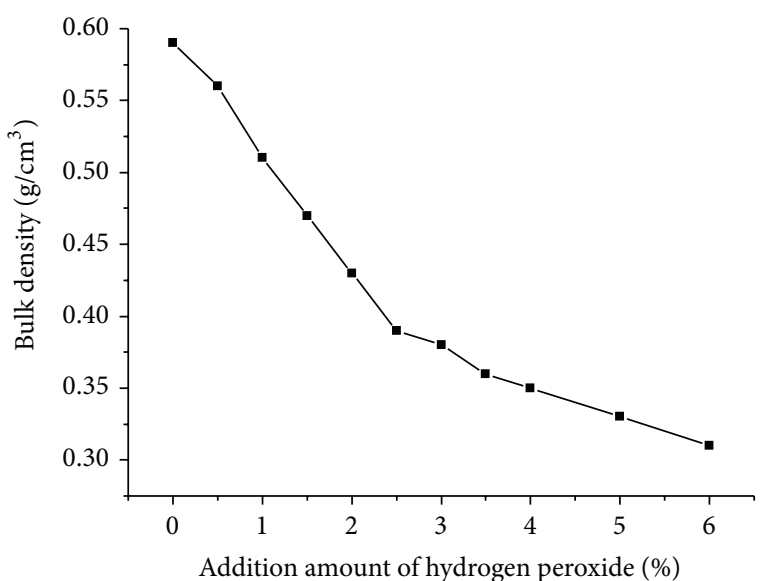

FIGURE 8: Variation of bulk density values of panel materials containing sepiolite nanofibers after adding different amounts of hydrogen peroxide.

panel materials [33-35]. Consequently, the hydrogen peroxide is added to the above mixture, which can be easily decomposed into water and oxygen and play an important role in the decrease of bulk density. Figure 8 shows the variation of bulk density values of panel materials containing sepiolite nanofibers after adding different amounts of hydrogen peroxide.

From Figure 8, it can be seen that the panel materials containing sepiolite nanofibers after adding different amounts of hydrogen peroxide have different bulk density values. Among them, the panel materials containing sepiolite nanofibers show the obvious decrease of bulk density after adding different amounts of hydrogen peroxide when the addition amount of sodium silicate is less than $2.5 \%$. When the addition amount of hydrogen peroxide is more than $2.5 \%$, the bulk density of panel materials containing sepiolite nanofibers decreases slowly, which can be explained as follows. When the addition amount of sodium silicate is less than $2.5 \%$, different contents of hydrogen peroxide enter structural unit of panel materials, which can decompose and release more and more oxygen rapidly in the heating process. Consequently, the bulk density of panel materials containing sepiolite nanofibers decreases significantly along with the increase of addition amount of hydrogen peroxide. When the addition amount of sodium silicate is more than $2.5 \%$, contents of oxygen tend to be saturated and agglomerating action increases, therefore the bulk density of panel materials containing sepiolite nanofibers decreases slowly. At the same time, the compressive strength value of panel materials treated by $2.5 \%$ of hydrogen peroxide is $0.39 \mathrm{MPa}$, which meets the requirement of GB 4272-92 and JGJ144-2008. According to the above analysis, $2.5 \%$ is selected as the optimal condition of addition amount of hydrogen peroxide value in order to obtain the thermal insulation inorganic panel. Thus, the optimal process of heat insulation materials performance is shown as follows: $120^{\circ} \mathrm{C}$ of drying temperature, $1.6 \%$ of sodium silicate as inorganic adhesive, $12 \%$ of sodium

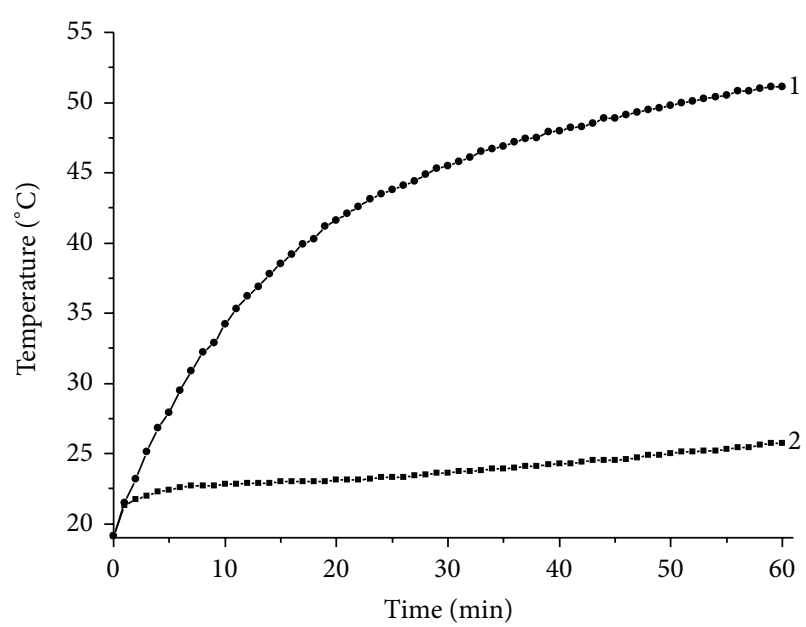

FIGURE 9: Thermal insulation effect of thermal insulation energy saving panel materials (1: upper air temperature; 2: lower air temperature).

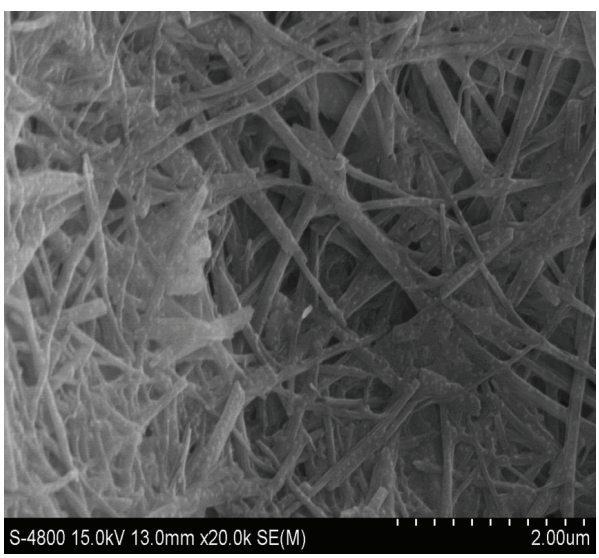

FIgURE 10: Cross-section microstructure of thermal insulation energy saving panel materials.

fluorosilicate as curing agent in sodium silicate, and $2.5 \%$ of hydrogen peroxide. The thermal insulation effect of thermal insulation energy saving panel materials is shown in Figure 9. From Figure 9, it can be seen that the lower air temperature is much lower than the upper one, which indicates that the panel materials as prepared could arrest heat transmission effectively.

Figure 10 shows the cross-section microstructure of thermal insulation energy saving panel materials. We can see from Figure 10 that the sepiolite nanofibers were well distributed and combined tightly with each other in the thermal insulation energy saving panel materials. The matching function of vacuum construction in the sepiolite nanofibers and void among sepiolite nanofibers could arrest heat conduction effectively. 


\section{Conclusions}

The thermal insulation energy saving panel materials containing sepiolite nanofibers with characteristics of high efficiency and low cost were prepared by the synergistic action of water soluble sodium silicate as inorganic adhesive, sodium fluorosilicate as curing agent, and hydrogen peroxide. The results showed that the thermal insulation energy saving panel materials as prepared with microporous and mesoporous structure have high compressive strength and low bulk density. The optimal process of heat insulation materials performance was as follows: $120^{\circ} \mathrm{C}$ of drying temperature, $1.6 \%$ of sodium silicate as inorganic adhesive, $12 \%$ of sodium fluorosilicate as curing agent in sodium silicate, and 2.5\% of hydrogen peroxide. Accordingly, the thermal insulation energy saving panel materials as prepared have good thermal insulation effect and high mechanical performance.

\section{Conflict of Interests}

The authors declare that there is no conflict of interests regarding the publication of this paper.

\section{Acknowledgments}

This research was financially supported by the Application Foundation and Advanced Technology Research Program of Tianjin, China (Grant no. 12JCQNJC02100) and the National Key Technology R\&D Program of China (Grant no. 2011BAE30B04).

\section{References}

[1] L. E. Mavromatidis, M. EL Mankibi, P. Michel, and M. Santamouris, "Numerical estimation of time lags and decrement factors for wall complexes including Multilayer Thermal Insulation, in two different climatic zones," Applied Energy, vol. 92, pp. 480-491, 2012.

[2] W. M. Lewandowski and W. L. Iwaniak, "The external walls of a passive building: a classification and description of their thermal and optical properties," Energy and Buildings, vol. 69, pp. 93-102, 2014.

[3] C. G. Yang, L. Xu, L. Q. Zhang, and N. Chen, "An adiabatic calorimeter for heat capacity measurements of polyurethane foam with blowing agent of HFC245fa in the temperature range 60-290K," Energy Conversion and Management, vol. 47, no. 910, pp. 1124-1132, 2006.

[4] L. Wang, Y. Wang, W. Q. Zhang et al., "Finite element simulation of stress distribution and development in 8YSZ and doubleceramic-layer $\mathrm{La}_{2} \mathrm{Zr}_{2} \mathrm{O}_{7} / 8 \mathrm{YSZ}$ thermal barrier coatings during thermal shock," Applied Surface Science, vol. 258, no. 8, pp. 3540-3551, 2012.

[5] L. H. Liang, H. Wei, X. C. Chang, W. Xu, X. N. Li, and Y. G. Wei, "Enhanced insulation temperature and the reduced thermal conductivity of nanostructured ceramic coating systems," International Journal of Heat and Mass Transfer, vol. 65, pp. 219224, 2013.
[6] A. Joly, P. Brun, J. Lacombe, G. Tricot, and A. Denoirjean, "Structural characterization of an electrically insulating diffusion barrier on a plasma-sprayed ceramic for severe environment applications," Surface and Coatings Technology, vol. 220, pp. 204-208, 2013.

[7] R. Ghasemi, R. S. Razavi, R. Mozafarinia, and H. Jamali, "Laser glazing of plasma-sprayed nanostructured yttria stabilized zirconia thermal barrier coatings," Ceramics International, vol. 39, no. 8, pp. 9483-9490, 2013.

[8] B. B. Ekici, A. A. Gulten, and U. T. Aksoy, "A study on the optimum insulation thicknesses of various types of external walls with respect to different materials, fuels and climate zones in Turkey," Applied Energy, vol. 92, pp. 211-217, 2012.

[9] L. Zhou, Z. Z. Jing, Y. Zhang, K. Wu, and E. H. Ishida, "Stability, hardening and porosity evolution during hydrothermal solidification of sepiolite clay," Applied Clay Science, vol. 69, pp. 30-36, 2012.

[10] S. M. Jin, K. X. Cui, H. Guan, M. Yang, L. Liu, and C. Lan, "Preparation of mesoporous MCM-41 from natural sepiolite and its catalytic activity of cracking waste polystyrene plastics," Applied Clay Science, vol. 56, pp. 1-6, 2012.

[11] M. Suarez and E. Garcia-Romero, "Variability of the surface properties of sepiolite," Applied Clay Science, vol. 67-68, pp. 7282, 2012.

[12] A. S. Özcan and Ö. Gök, "Structural characterization of dodecyltrimethylammonium (DTMA) bromide modified sepiolite and its adsorption isotherm studies," Journal of Molecular Structure, vol. 1007, pp. 36-44, 2012.

[13] H. Chen, H. Lu, Y. Zhou, M. Zheng, C. Ke, and D. Zeng, "Study on thermal properties of polyurethane nanocomposites based on organo-sepiolite," Polymer Degradation and Stability, vol. 97, no. 3, pp. 242-247, 2012.

[14] Y. B. Sun, G. H. Sun, Y. M. Xu et al., "In situ stabilization remediation of cadmium contaminated soils of wastewater irrigation region using sepiolite," Journal of Environmental Sciences, vol. 24, no. 10, pp. 1799-1805, 2012.

[15] M. Uurlu and M. H. Karaolu, "Adsorption of ammonium from an aqueous solution by fly ash and sepiolite: isotherm, kinetic and thermodynamic analysis," Microporous and Mesoporous Materials, vol. 139, no. 1-3, pp. 173-178, 2011.

[16] C. Cheng, L. Ma, J. Ren et al., "Preparation of polyethersulfonemodified sepiolite hybrid particles for the removal of environmental toxins," Chemical Engineering Journal, vol. 171, no. 3, pp. 1132-1142, 2011.

[17] R. Pina-Zapardiel, A. Esteban-Cubillo, J. F. Bartolome, C. Pecharroman, and J. S. Moya, "High wear resistance white ceramic glaze containing needle like zircon single crystals by the addition of sepiolite $\mathrm{n}-\mathrm{ZrO}_{2}$," Journal of the European Ceramic Society, vol. 33, pp. 3379-3385, 2013.

[18] A. M. Sevim, R. Hojiyev, A. Gül, and M. S. Çelik, "An investigation of the kinetics and thermodynamics of the adsorption of a cationic cobalt porphyrazine onto sepiolite," Dyes and Pigments, vol. 88, no. 1, pp. 25-38, 2011.

[19] B. F. Jones and K. M. Conko, "Environmental influences on the occurrences of sepiolite and palygorskite: a brief review," Developments in Clay Science, vol. 3, pp. 69-83, 2011.

[20] E. Garcia-Romero and M. Suarez, "Sepiolite-palygorskite: textural study and genetic considerations," Applied Clay Science, vol. 86, pp. 129-144, 2013.

[21] F. Wang, J. Liang, Q. Tang, L. Li, and L. Han, "Preparation and far infrared emission properties of natural sepiolite nanofibers," 
Journal of Nanoscience and Nanotechnology, vol. 10, no. 3, pp. 2017-2022, 2010.

[22] H. Yin, J.-S. Liang, Q.-G. Tang, G.-C. Liang, L.-J. Wang, and G.-S. Li, "Influence of sepiolite microstructure on thermal insulation properties of coating material," Journal of Synthetic Crystals, vol. 34, no. 3, pp. 519-524, 2005.

[23] A. Miura, K. Nakazawa, T. Takei et al., "Acid-, base-, and heatinduced degradation behavior of Chinese sepiolite," Ceramics International, vol. 38, pp. 4677-4684, 2012.

[24] S. Tunç, O. Duman, and A. Çetinkaya, "Electrokinetic and rheological properties of sepiolite suspensions in the presence of hexadecyltrimethylammonium bromide," Colloids and Surfaces A, vol. 377, no. 1-3, pp. 123-129, 2011.

[25] E. H. Duan, J. Han, Y. Song et al., "Adsorption of styrene on the hydrothermal-modified sepiolite," Materials Letters, vol. 111, pp. 150-153, 2013.

[26] E. Eren, H. Gumus, and A. Sarihan, "An investigation of the catalytic decomposition of formic acid on raw and manganese oxide coated sepiolite surfaces," Applied Clay Science, vol. 62-63, pp. 1-7, 2012.

[27] Q. G. Tang, J. S. Liang, G. C. Liang, and F. Wang, "Equipment for checking-out effect of heat insulation for building material, checking-out method and evaluation method," CN 200610015611. 9A, 2007.

[28] H. Jin, E. A. Prasetyanto, N. Jiang, S.-M. Oh, and S.-E. Park, "Length dependency of hydrocarbon adsorption on nanostacked MFI zeolite by tracer chromatography," Applied Surface Science, vol. 256, no. 17, pp. 5508-5512, 2010.

[29] R. Sako and J. Sakai, "Effect of curing temperature on coating structure and corrosion resistance of ammonium zirconium carbonate on galvanized steel surface," Surface and Coatings Technology, vol. 219, pp. 42-49, 2013.

[30] Y. Zhang, D. Wang, and G. Zhang, "Photocatalytic degradation of organic contaminants by $\mathrm{TiO}_{2} /$ sepiolite composites prepared at low temperature," Chemical Engineering Journal, vol. 173, no. 1, pp. 1-10, 2011.

[31] S. Tunç, O. Duman, and B. Kanci, "Rheological measurements of Na-bentonite and sepiolite particles in the presence of tetradecyltrimethylammonium bromide, sodium tetradecyl sulfonate and Brij 30 surfactants," Colloids and Surfaces A, vol. 398, pp. 37-47, 2012.

[32] B. F. Jones and K. M. Conko, "Environmental influences on the occurrences of sepiolite and palygorskite: a brief review," Developments in Clay Science, vol. 3, pp. 69-83, 2011.

[33] Q. Chen, S. Wang, and Z. Li, "Fabrication and characterization of aluminum silicate fiber-reinforced hollow mesoporous silica microspheres composites," Microporous and Mesoporous Materials, vol. 152, pp. 104-109, 2012.

[34] N. Du, J. Fan, H. Wu, and W. Sun, "Optimal porosity distribution of fibrous insulation," International Journal of Heat and Mass Transfer, vol. 52, no. 19-20, pp. 4350-4357, 2009.

[35] R. Arambakam, H. V. Tafreshi, and B. Pourdeyhimi, "A simple simulation method for designing fibrous insulation materials," Materials and Design, vol. 44, pp. 99-106, 2013. 

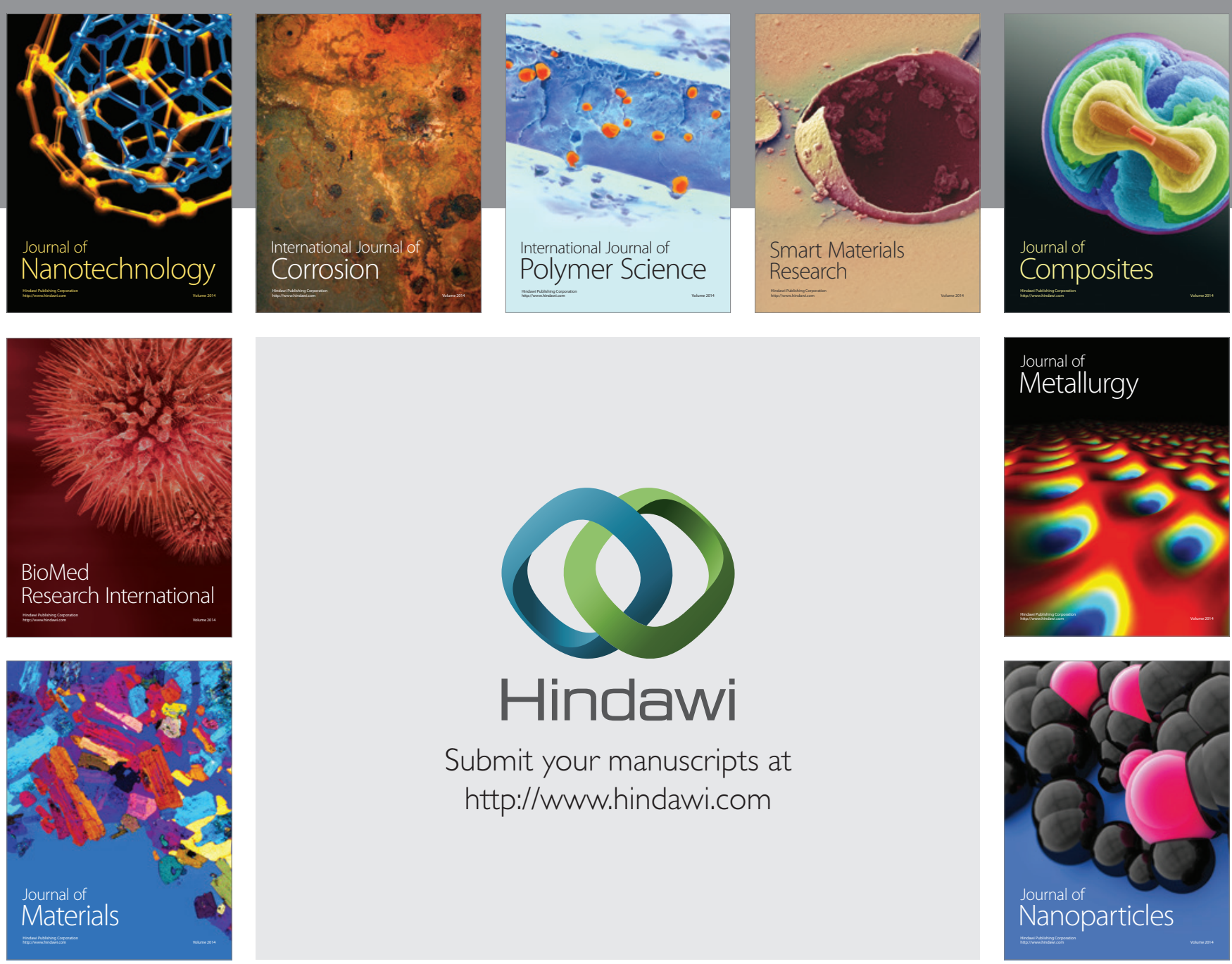

Submit your manuscripts at http://www.hindawi.com
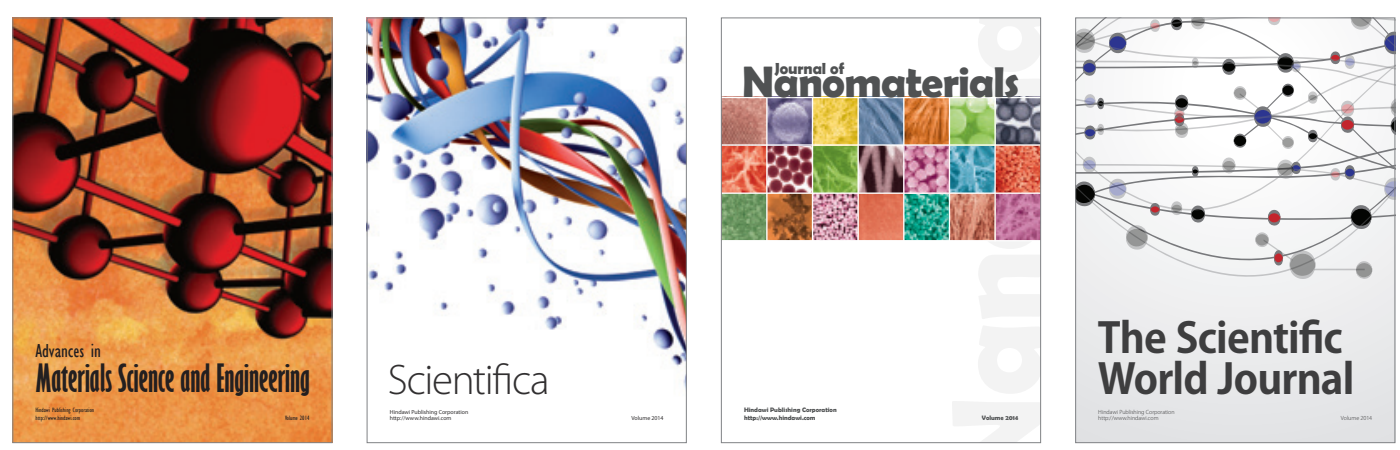

\section{The Scientific World Journal}
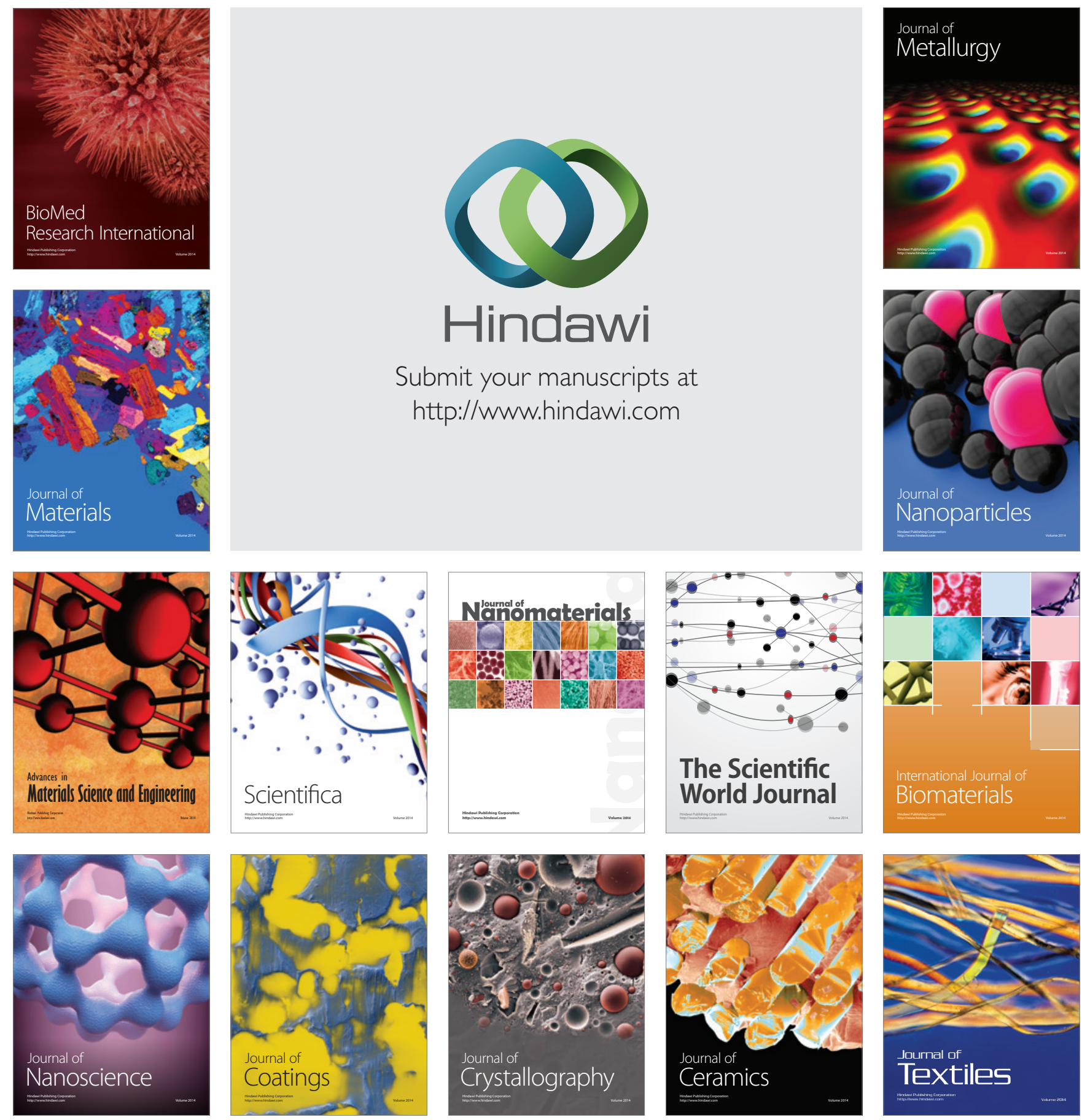